\title{
Numerical Simulation of the Starting Flow Down a Step
}

\author{
Rodrigue Savoie, Yves Gagnon \\ Université de Moncton, Campus d'Edmundston \\ Edmundston (NB), Canada
}

\author{
Yves Mercadier \\ Département de génie mécanique \\ Université de Sherbrooke \\ Sherbrooke (Qc), Canada
}

\begin{abstract}
The starting flow down a step in an open channel is simulated using a vortex method. A mixed Eulerian-Lagrangian scheme based on the vortex-in-cell algorithm and random walk diffusion is used with a non-uniform mesh. The comparison of numerical and experimental instantaneaous streamline plots show good agreement, especially for early stages of the flow development. It is also shown that, during intermediate stages of the flow development and for the range of Reynolds numbers investigated, the distance from the step to the reattachment point of the main vorticity domain increases linearly with the dimensionless time. Again, good agreement is obtained between experimental data and computed solutions.
\end{abstract}

\section{Introduction}

The random vortex method (RVM), proposed by Chorin [4], was initially developed as a grid-free, full-Lagrangian method. In the RVM, the vorticity field is discretized among $N$ vortex elements in which the vorticity is distributed according to a radially symmetric core function. The dynamics of the vorticity field is governed by the vorticity transport equation which is solved in two fractional steps: a convection step and a diffusion step. In the convection step, the velocity of every vortex element is obtained from the vorticity field while diffusion is simulated by performing random walk movements on the vortex elements.

In full-Lagrangian schemes, the potential boundary condition of the flow is implemented using the image system of the vortex elements while the induced velocity field is computed from the Biot-Savart law, see for example, Ghoniem et al. [9], Ghoniem and Gagnon [11] and Gagnon et al. [8]. However, because of the $O\left(N^{2}\right)$ operation count of the Biot-Savart law, the computational time can be relatively important.

On the other hand, Christiansen [5] presented a mixed Eulerian-Lagrangian vortex method where the velocity induced by the vorticity field on the Lagrangian vortex elements is obtained from an Eulerian velocity field. The vortex-in-cell (VIC) algorithm is used in the coupling of the Eulerian approach and the Lagrangian scheme. This mixed Eulerian-Lagrangian scheme, with an $O(N+P \log P)$ operation count ( $P$ being the number of points on the mesh), is less computationally intensive than the corresponding full-Lagrangian scheme and is more easily extendable to complex geometries.

Using a mixed Eulerian-Lagrangian scheme, Smith and Stansby [18] simulated the impulsively started flow around a circular cylinder. The numerical results are compared with analytical solutions for shorter times and with accurate Eulerian numerical simulations and experimental flow visualizations for longer times. The agreement is good when a sufficiently large number of vortex 
elements is introduced at every timestep. The number required increases as the Reynolds number increases.

More recently, Stansby and Slaouti [19] presented converged simulations of vortex shedding from a circular cylinder including the influence of blockage using the VIC algorithm and an overlapping system of rectangular and polar meshes. Closer to the work of this paper, Gagnon and Huang [7] presented the results of two-dimensional simulations of flows inside channels with and without injection of a secondary flow and for flows at both low and high Reynolds numbers. A non-uniform rectangular mesh was used in their Eulerian computations. Finally, Westermann [20] extended the algorithm to boundary-fitted meshes with moving boundaries.

In many situations fluid flows can be considered two-dimensional before the onset of threedimensional instabilities which occur at a time that depends on the Reynolds number. Such situations allow for accurate numerical convergence checks of two-dimensional algorithms. The laminar starting flow down a step is a well defined unsteady flow which can serve in a convergence check of a two-dimensional numerical algorithm. While most backward-facing step flows are studied experimentally in confined geometries and for the mean and stationary characteristics (see, for example, the experimental works of de Brederode and Bradshaw [3], Denham and Patrick [6], Pitz and Daily [16], Armaly et al. [2], Adams and Jonhston [1], Isomoto and Honami [15] and Hasan [12]), Honji [13] studied the laminar starting flow down an unconfined step. The experiment was made by establishing a steady motion of a test-body inside a water tank whose width was very large in comparison to the height of the step (75:1.85). The Reynolds number of the flows (based on the step height and the towing speed of the body) were less than 500. Aluminum-dust and electrochemical methods were used as flow visualization techniques. It was observed that the flows were fairly two-dimensional over the range of Reynolds numbers studied.

Honji's experiments provide extensive data for the fine structure of the time-dependent flow down an unconfined step and thus constitutes an excellent test-case for the numerical algorithm. Figure 1 presents the geometry simulated, with the flow being from left to right. The dimensions correspond and are in relation to the physical dimensions of the experimental work of Honji [13].

The formulation of the problem and a brief description of the algorithm are presented in the next section, followed by comparisons of numerical results with experimental results. Details on the algorithm and more results are presented in Savoie et al. [17]. A numerical convergence check of the algorithm is also presented.

\section{Formulation}

\subsection{Formulation}

The non-dimensional equations that govern viscous incompressible flows are the continuity equation and the Navier-Stokes equations, respectively:

$$
\begin{gathered}
\nabla \cdot \mathbf{u}=0 \\
\frac{\partial \mathbf{u}}{\partial t}+(\mathbf{u} \cdot \nabla)=-\nabla p+\frac{1}{R e} \nabla^{2} \mathbf{u}
\end{gathered}
$$

where $\mathbf{u}=(u, v)$ is the velocity vector (streamwise $x$ and cross-stream $y$ components, respectively), $p$ is the pressure, $t$ is the time and $R e$ is the Reynolds number defined as $R e=\rho U H / \mu$ where the reference velocity $U$ is the uniform inlet velocity, $H$ is the height of the step, $\rho$ is the density and $\mu$ is the dynamic viscosity.

The boundary conditions for the geometry on Fig. 1 include the no-slip condition on the lower upstream wall, the lower downstream wall and the vertical step wall, i.e. $\mathbf{u}=(u, v)=(0,0)$. A 
uniform velocity $U$ is imposed at the inlet section of the computational domain, while a zero crossstream velocity, i.e. $v=0$, is imposed at the oulet section. For the top boundary condition, a slip wall is assumed for computational purposes. This implies not only that $v=0$ but also that no vorticity is to be generated on this wall. A discussion on the choice and the effects of the boundary conditions is presented in Savoie et al. [17]. Potential flow is assumed as the initial condition of the time-dependent simulations.

Using the definition of vorticity, in two dimensions,

$$
\omega=|\nabla \times \mathbf{u}|
$$

the problem is transformed into vorticity variables. The result is the streamfunction-vorticity $(\psi-\omega)$ relation:

$$
\nabla^{2} \psi=-\omega
$$

and the two-dimensional vorticity transport equation:

$$
\frac{\partial \omega}{\partial t}+(\mathbf{u} \cdot \nabla) \omega=\frac{1}{R e} \nabla^{2} \omega
$$

The distribution of vorticity at time $t$ is approximated by $N$ point-wise singular vortex elements of circulation $\Gamma_{i}$ :

$$
\omega=\sum_{i=1}^{N} \Gamma_{i} \delta\left(\mathbf{x}-\mathbf{x}_{i}\right)
$$

where $\mathbf{x}_{i}$ is the position of vortex element $i$ and $\delta(\mathbf{x})$ is the Dirac delta function.

\subsection{Transport of vorticity}

The vorticity transport equation, Eq. 5, is solved in two fractional steps (Chorin [4]): a convective transport of vorticity and a diffusive transport of vorticity, respectively:

$$
\frac{\partial \omega}{\partial t}+(\mathbf{u} \cdot \nabla) \omega=0
$$

and

$$
\frac{\partial \omega}{\partial t}=\frac{1}{R e} \nabla^{2} \omega
$$

The convective transport of vorticity in Lagrangian form is governed by a simple first order ordinary differential equation where the vortex elements are convected in the velocity field while they preserve their individual circulation. On the other hand, diffusion is simulated stochastically by performing random walk movements on the vortex elements (Chorin [4], Ghoniem and Sherman $[10])$.

The convection velocity of the vortex elements induced by the vorticity field and the satisfaction of the boundary conditions is obtained on a non-uniform mesh, as described in the following subsection.

\subsection{Non-uniform Eulerian mesh}

The flow field simulated is characterized by different length scales as a function of the position. The flow near the walls are characterized by small structures inside the boundary layers. The shear layer formed at the separation point of the flow and the recirculation zone are also characterized by small scale structures. Also, the flow on the bottom wall at the inlet section is characterized by sharp 
gradients. Therefore, the mesh of the computational domain is refined in these areas. Elsewhere in the computational domain, a coarser mesh may be used without affecting the accuracy of the solution, while allowing for a more efficient solution (Smith and Stansby [18], Gagnon and Huang $[7])$.

The governing equations of the fluid flow problem are transformed in the general coordinates:

$$
\xi=\xi(x) \quad \text { and } \quad \eta=\eta(y)
$$

and Eq. 4 transforms to:

$$
\frac{1}{x_{\xi}^{2}} \frac{\partial^{2} \psi}{\partial \xi^{2}}+\frac{1}{y_{\eta}^{2}} \frac{\partial^{2} \psi}{\partial \eta^{2}}-\frac{x_{\xi \xi}}{x_{\xi}^{3}} \frac{\partial \psi}{\partial \xi}-\frac{y_{\eta \eta}}{y_{\eta}^{3}} \frac{\partial \psi}{\partial \eta}=-\omega
$$

Using an iterative technique, Eq. 10 is solved for y on the transformed mesh by alloting the circulation of the Lagrangian vortex elements between the four corner nodes of a cell as per the VIC algorithm (Christiansen [5]). The two components of velocity, $u$ and $v$, are then computed at every mesh point using the following expressions in the general coordinates system:

$$
\begin{gathered}
u=\frac{1}{x_{\xi}} \frac{\partial \psi}{\partial \xi} \\
v=-\frac{1}{y_{\eta}} \frac{\partial \psi}{\partial \eta}
\end{gathered}
$$

In order to satisfy the constraints imposed by the presence of small scale structures, algebraic transformations were chosen to ensure high mesh densities in the critical regions listed above.

In the $y$ direction, two computational domains are used: the flow domain below the height of the upstream horizontal wall and the flow domain above the height of the upstream horizontal wall. For the lower domain, the transformations are:

$$
\begin{gathered}
\eta_{s}=-1+\frac{2 j}{j_{\text {step }}} \quad j=0 \rightarrow j_{\text {step }} \\
y=y_{\text {min }}+\left(y_{\text {step }}-y_{\text {min }}\right)\left(\frac{1}{2}+\frac{\tanh \left(\eta_{s} \alpha_{y}\right)}{2 a_{y}}\right)
\end{gathered}
$$

where

$$
\alpha_{y}=\tanh ^{-1}\left(a_{y}\right)
$$

and where $a_{y}$ is the mesh density factor. Here, $j_{\text {step }}=j_{\max } / 2$ where $j_{\max }$ is the total number of mesh points in the cross-stream direction.

For the domain above the height of the upstream horizontal wall, the transformations are:

$$
\begin{gathered}
\eta_{n}=\frac{j-j_{\text {step }}}{j_{\text {max }}-j_{\text {step }}} \quad j=j_{\text {step }} \rightarrow j_{\max } \\
y=y_{\text {step }}+b_{y}\left(e^{\eta_{n} c_{y}}-1\right)
\end{gathered}
$$

Here, $b_{y}$ and $c_{y}$ are chosen to ensure that the mesh densities are equal above and below the position of the upstream horizontal wall. Therefore,

$$
\frac{e^{c_{y}}-1}{e^{c_{y} /\left(j_{\max }-j_{\mathrm{step}}\right)}-1}=\frac{y_{\mathrm{max}}-y_{\mathrm{step}}}{y_{\mathrm{step}}-y_{j_{\mathrm{step}}-1}}
$$


and

$$
b_{y}=\frac{y_{\max }-y_{\text {step }}}{e^{c_{y}}-1}
$$

In this study, $y_{\min }=-1, y_{\mathrm{step}}=0$ and $y_{\max }=19$. The value of $j_{\max }$ ranged from 200 to 400 .

A similar transformation as the latter one was used in the streamwise $(x)$ direction. For results presented in this study, $x_{\min }=-25, x_{\text {step }}=0$ and $x_{\max }=25$ while the value of $i_{\max }$ ranged from 500 to 1000 .

\subsection{Vorticity generation on solid walls}

On solid walls, the satisfaction of the potential boundary condition does not necessarily satisfy the zero tangential velocity condition of the velocity vector. Circulation is thus generated at every timestep, mimicking the creation of vorticity due to the action of viscosity on the boundaries (Chorin [4]). The circulation created at wall-point $i$ to annihilate the slip velocity at this point is obtained from the definition of circulation:

$$
\Gamma_{\text {wall }, i}=\int \mathbf{u}_{\text {slip }} \cdot d \mathbf{s}
$$

where point $i$ is located in the middle of adjoining mesh points defining a boundary element. The integral is performed on the boundary element of point $i$, up to the neighbouring mesh points, while $\mathbf{u}_{\text {slip }}$ is obtained from the average velocities of the mesh points defining the boundary element and $d \mathbf{s}$ is the length of the boundary element. The circulation created for each boundary element, at every timestep, is divided among Nnew elements according to the "minimum generation algorithm" (Savoie et al. [17]):

$$
N_{\text {new }}=\max \left[\operatorname{int}\left(\frac{\Gamma_{\text {wall }, i}}{\Gamma_{\text {opt }}}+0.5\right), n_{\text {min }}\right]
$$

where $\Gamma_{\text {opt }}$ is an optimum value of circulation of the vortex elements (Ghoniem and Gagnon [11]) and $n_{\min }$ is a minimum number of vortex elements created on a boundary element at every timestep. The circulation generated is divided equally among the newly created elements which are evenly spaced along the length of the boundary element. The resulting flow field, consisting of the newly created and the existing vortex elements, will evolve according to the vorticity transport equation.

\section{Results}

Results presented in this paper were obtained with the parameter values $\Gamma_{\text {opt }}=10^{-4}, n_{\min }=5$ and a timestep of $\Delta t=0.05$ while a second-order modified Euler method is used in the convection of the vortex elements. The non-uniform mesh consisted of 501 mesh points in the streamwise direction and 201 mesh points in the cross-stream direction with density factors $a_{x}=a_{y}=0.95$. Specific individual influences of these parameters are discussed in Savoie et al. [17].

The experimental images of Honji [13] were made at times of observation refering to the midtime of exposure (ranging from 0.4 to 8 seconds) since the start of the flow. The instantaneous numerical results refering to the instantaneous times of Honji are thus averaged over a few timesteps of the simulations, mimicking a short time exposure of the physical flow. This has the advantage of having smoother numerical instantaneous streamline plots without changing their general shape.

In the very early development, the flow separates from the upper corner of the step and the reattachment point on the vertical wall moves down from the upper corner to the lower corner of the step in a time of approximately 0.7 (Savoie et al. [17]). When the reattachment point reaches the 
lower corner of the step, it moves downstream on the bottom wall. The length of the recirculation zone increases linearly until the flow reaches its stationary dimension. This behaviour was observed on all test-cases, irrespective of the Reynolds numbers.

A comparison of instantaneous streamline plots of experimental visualizations for a flow with a Reynolds number of 99.6 and the numerical visualizations for a flow with $R e=100$ is presented for different times on Fig. 2. The global comparison is excellent and the dynamical behaviour of the flow is accurately simulated. At an early time $(t=5.10)$, the recirculation zone is formed of one large vorticity domain. At an intermediate time $(t=12.40)$, the vorticity domain tends to separate from the step, without actually separating. At later times $(t=41.70)$, the numerical recirculation zone is formed of one vorticity domain, but with two center of rotations in comparison to the single center in the experimental flow. This difference can be attributed to a long time-exposure for the experimental image in comparison to the very short "time-exposure" for the numerical results.

The flow with a Reynolds number of 300 is characterized by the development of four vorticity domains inside the recirculation zone. The numerical visualizations are compared on Fig. 3 to the experimental visualizations for a Reynolds number of 304. At $t=3.85$, the recirculation zone is composed of one large coherent eddy forming the main vorticity domain. At $t=10.5$, the recirculation zone is composed of three vorticity domains. At later times, $t=23.8$, the main vorticity domain is detached considerably from the step and no definite structure is present near the step wall.

An average streamline plot was computed by averaging the instantaneous numerical flow fields and is compared on Fig. 4 to the corresponding experimental visualization. The time-exposure of the experimental flow was 30 seconds, with the time indicated refering to the middle of the time-exposure with respect to the begining of the flow (Honji [14]). For this average flow, the recirculation zone is formed of one large vorticity domain.

Finally, the reattachment point as a function of time is presented on Fig. 5 where the hollow symbols are for the experimental results of Honji [13] and the filled symbols depict the numerical results for different Reynolds numbers. In the latter case, the length of the recirculation zone was inferred from the position of reattachment of the dividing streamline. For all cases, the evolution of the reattachment point obtained from the numerical results is in very good agreement with the experimental evolution. The linear increase in the position of the reattachment point is clearly seen both from the experimental results and numerical results.

\section{Conclusion}

Numerical simulations of the laminar starting flow down a step were presented and compared to experimental flow visualizations. The numerical results have confirmed that the flow regime is a strong function of the Reynolds number, most particularly for the structure of the separated region. A more detailled discusion of the results and the influence of the numerical parameters are presented in Savoie et al. [17].

The starting flow down an unconfined step has proven to be an important test for the mixed Eulerian-Lagrangian scheme with non-uniform meshes used in this work. This study allows for a better understanding of the fundamental modelling of fluid flows. Furthermore, by performing accurate unsteady simulations, insights on the dynamics of the flow are obtained. 


\section{Acknowledgements}

This work was supported by the Natural Sciences and Engineering Research Council (NSERC) of Canada and by the Faculté des études supérieures et de la recherche of the Université de Moncton.

\section{References}

[1] Adams, E.W. and Johnston, J.P., "Effects of the Separating Shear Layer on the Reattachment Flow Structure. Part 2: Reattachment Length and Wall Shear Stress," Exp. Fluids, 6, p. 493, 1988 .

[2] Armaly, B.F., Durst, F., Pereira, J.C.F. and Schonung, B., "Experimental and Theoretical Investigation of Backward-Facing Step Flow," J. Fluid Mech., 127, p. 473, 1983.

[3] Brederode, V. de and Bradshaw, P., "Three-Dimensional Flow in Nominally Two-Dimensional Separation Bubbles I: Flow Behind Rearward-Facing Step," Aero Rep. 72-19, Imp. College of Sc. and Tech., London, England, 1972.

[4] Chorin, A.J., "Numerical Study of Slightly Viscous Flow," J. Fluid Mech., 57, p. 785, 1973.

[5] Christiansen, J.P., "Vortex Methods for Flow Simulation," J. Comp. Phys., 13, p. 363, 1973.

[6] Denham, M. and Patrick, M., "Laminar Flow Over a Downstream-Facing Step in a TwoDimensional Flow Channel," Trans. Inst. of Chemical Eng'n, 52, p. 361, 1974.

[7] Gagnon, Y. and Huang, W., "Fast Vortex Method for the Simulation of Flows Inside Channels With and Without Injection," J. Thermal Sc., 2, p. 1, 1993.

[8] Gagnon, Y., Giovannini, A. and Hébrard, P., "Numerical Simulation and Physical Analysis of High Reynolds Number Recirculating Flows Behind Sudden Expansions," Phys. Fluids A, 5, p. 2377, 1993.

[9] Ghoniem, A.F., Chorin, A.J. and Oppenheim, A.K., "Numerical Modelling of Turbulent Flow in a Combustion Tunnel," Phil. Trans. R. Soc. Lond. A, 304, p. 303, 1982.

[10] Ghoniem, A.F. and Sherman, F.S., "Grid-Free Simulation of Diffusion Using Random Walk Methods," J. Comp. Phys., 61, p. 1, 1985.

[11] Ghoniem, A.F. and Gagnon, Y., "Vortex Simulation of Laminar Recirculating Flow," J. Comp. Phys., 68, p. 346, 1987.

[12] Hasan, M.A.Z., "The Flow Over a Backward-Facing Step Under Controlled Perturbation: Laminar Separation," J. Fluid Mech., 238, p. 73, 1992.

[13] Honji, H., "The Starting Flow Down a Step," J. Fluid Mech., 69, p. 229, 1975.

[14] Honji, H., Private communication, 1995.

[15] Isomoto, K. and Honami, S., "The Effect of Inlet Turbulence Intensity on the Reattachment Process Over a Backward-Facing Step," J. Fluids Eng'n, 111, p. 87, 1989.

[16] Pitz, R.W. and Daily, J.W., "An Experimental Study of Combustion: The Turbulent Structure of a Reacting Shear Layer Formed at a Rearward-Facing Step," NASA Cont. Rep. 165427, 1981. 
[17] Savoie, R., Gagnon, Y. and Mercadier, Y., "The Starting Flow Down a Step by a Vortex Method," submitted for publication, 1995.

[18] Smith, P.A. and Stansby, P.K., "Impulsively Started Flow Around a Circular Cylinder by the Vortex Method," J. Fluid Mech., 194, p. 45, 1988.

[19] Stansby, P.K. and Slaouti, A., "Simulation of Vortex Shedding Including Blockage by the Random-Vortex and Other Methods," Int'l. J. Num. Meth. Fluids, 17, p. 1003, 1993.

[20] Westermann, T., "Particle-In-Cell Simulations with Moving Boundaries-Adaptive Mesh Generation," J. Comp. Phys., 114, p. 161, 1994.

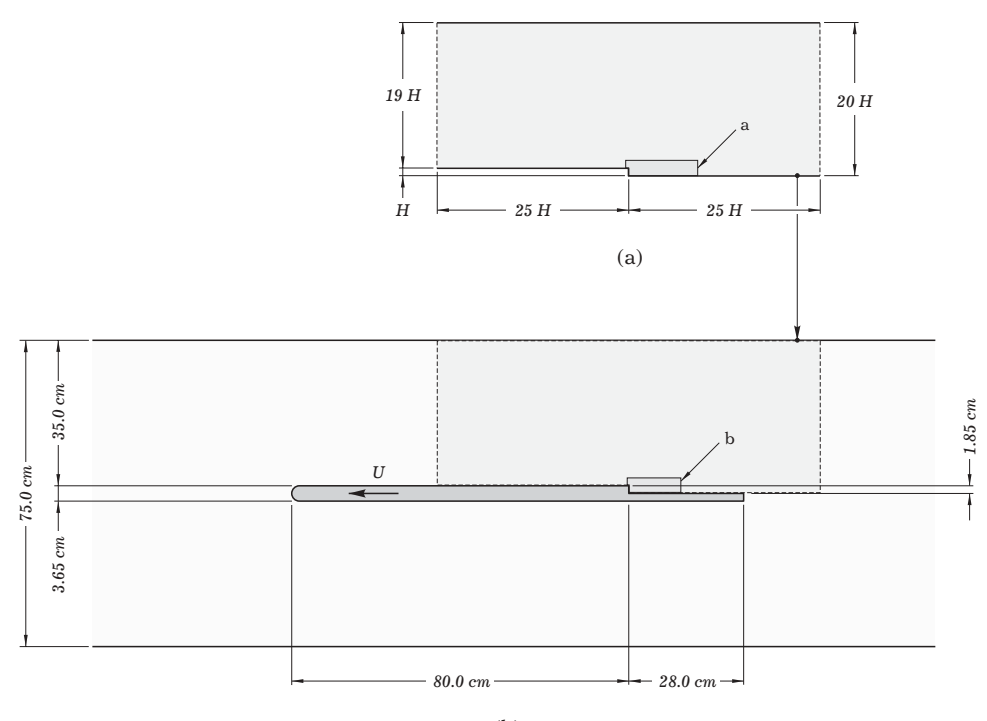

(b)

Figure 1: Model of the geometry. a: visualization window for Fig. 4. b: visualization window for Figs. 2, 3 . 


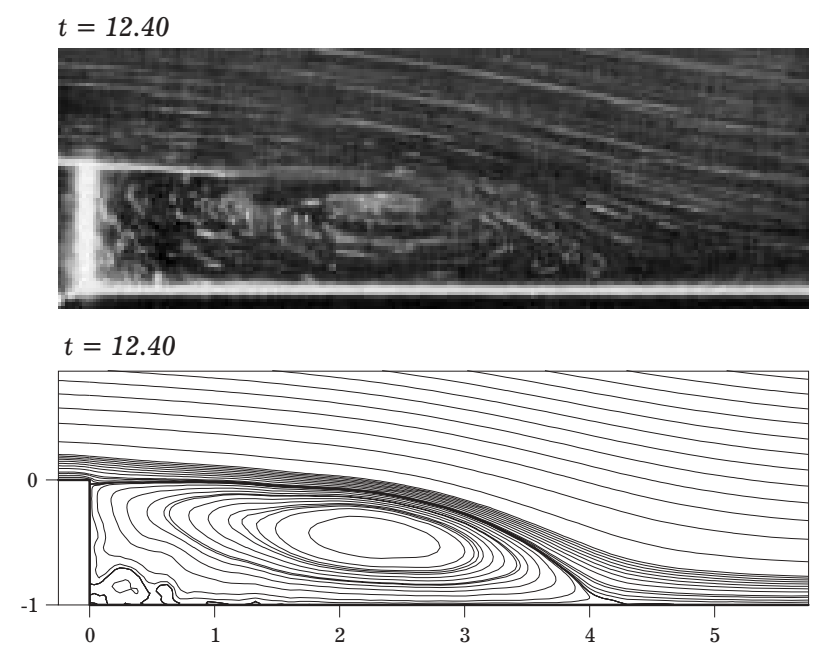

Figure 2: Comparison of the experimental visualizations and the numerical visualizations of instantaneous streamline plots, $R e=100$. Experimental images from Honji [13].
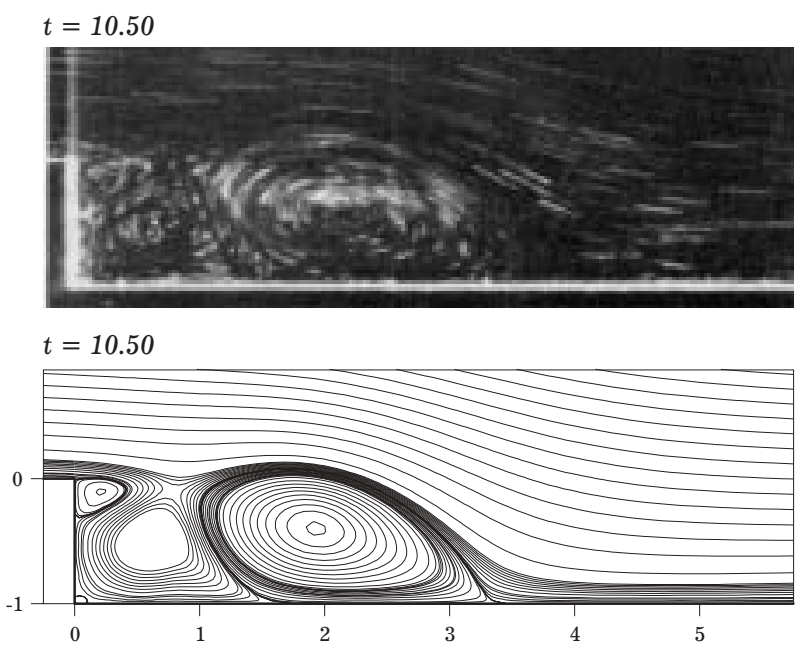

Figure 3: Comparison of the experimental visualizations and the numerical visualizations of instantaneous streamline plots, $R e=150$. Experimental images from Honji [13]. 

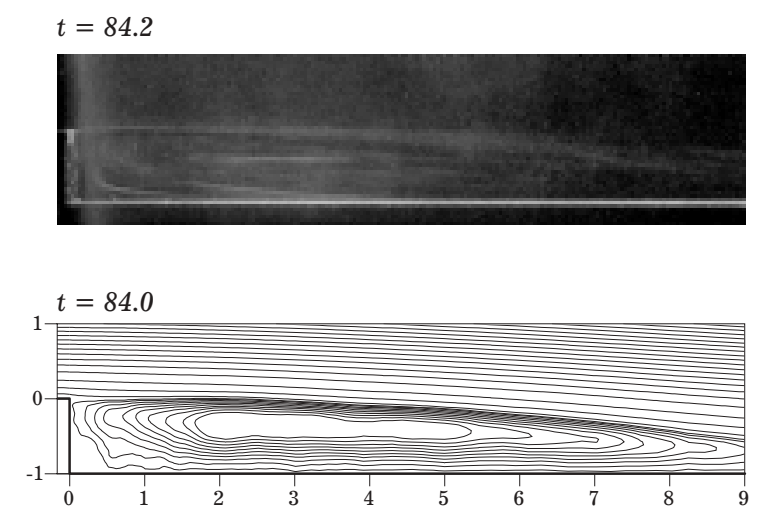

Figure 4: Comparison of the experimental visualizations and the numerical visualizations of instantaneous streamline plots, $R e=300$. Experimental image from Honji [13].

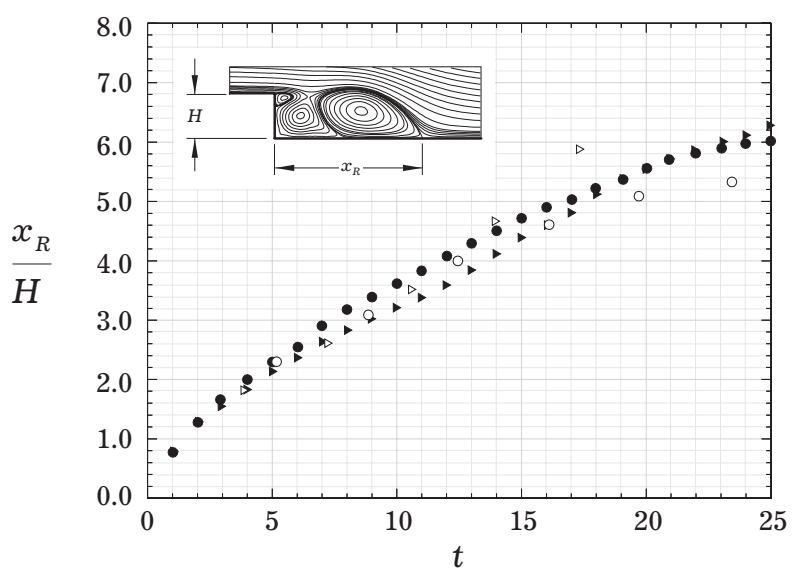

Figure 5: Comparison of the experimental and the numerical steady recirculation zone, $R e=300$. Experimental images from Honji [13] 